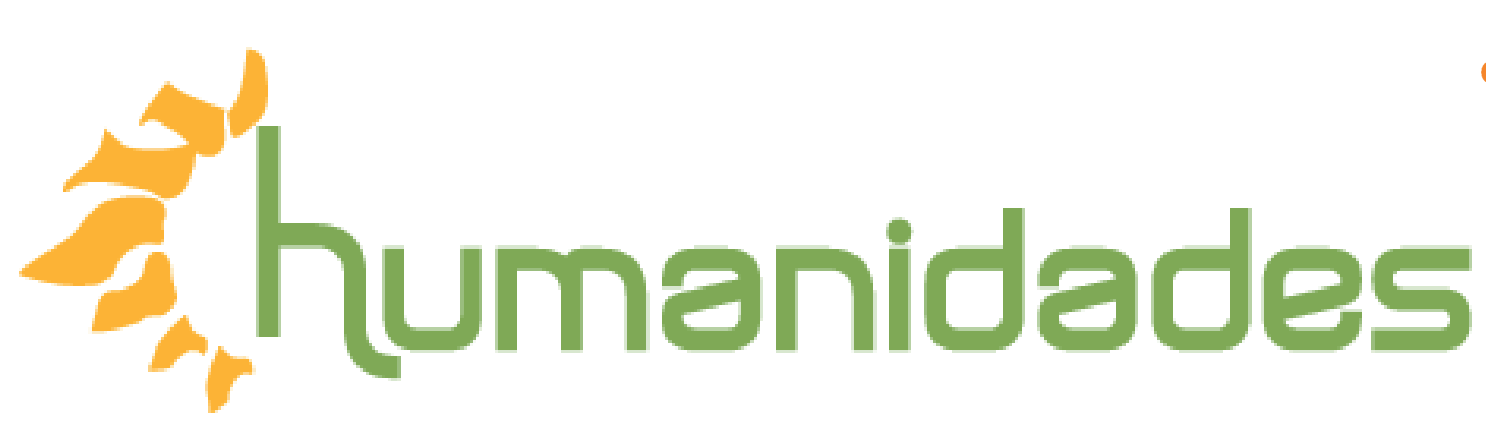

Revista de la Escuela de Estudios Generales, Universidad de Costa Rica

Enero-junio, 2018 •Volumen 8, número 1 • EISSN 2215-3934 •pp.51-82.

Recibido: 18-Septiembre-2017 Aceptado: 05-Diciembre-2017

\title{
La incorporación de la cultura y el patrimonio en el desarrollo sostenible: desafíos y posibilidades
}

DOI: http://dx.doi.org/10.15517/h.v8i1.31465

\section{Máster Bárbara Amanda Molina Neira}

Máster en Gestión del Patrimonio Cultural y Museología. Universidad de Barcelona, Facultad de Geografía e Historia, España.

baramonei@yahoo.com

Todos los derechos reservados. Universidad de Costa Rica. Esta revista se encuentra licenciada con Creative Commons. Reconocimiento-NoComercial-SinObraDerivada 3.0 Costa Rica. Correo electrónico: humanidades@ucr.ac.cr / Sitio web: http://revistas.ucr.ac.cr/index.php/ humanidades 


\title{
La incorporación de la cultura y el patrimonio en el desarrollo sostenible: desafíos y posibilidades
}

\section{Resumen}

El desarrollo sostenible es un modelo fundamental dentro de las agendas políticas actuales al proponer un equilibrio entre economía, sociedad y medio ambiente para lograr el bienestar. En este paradigma, la cultura ha sido relegada a pesar de sus contribuciones. Este artículo analiza el intento de incorporar la cultura y el patrimonio en la sostenibilidad para el desarrollo humano. Los hallazgos revelan que dicha integración ha sido compleja debido a factores semánticos, políticos y metodológicos. Esto implicaría que la superación de las dificultades depende del uso ético del concepto de desarrollo sostenible, de la voluntad política de las naciones a escala global y de la generación de metodologías coherentes con las diferentes realidades. El artículo proporciona así un marco para reflexionar sobre las posibilidades y limitaciones de la sostenibilidad cultural.

\section{Mainstreaming culture and cultural heritage into sustai- nable development: challenges and opportunities}

\begin{abstract}
Sustainable development is a fundamental model within the current political agendas as it proposes a balance between economy, society and environment to achieve welfare. In this paradigm, culture has been relegated despite its contributions. This article analyses the attempt to incorporate culture and cultural heritage into sustainability for human development. The findings reveal that such integration has been complex due to semantic, political and methodological factors. This would imply that overcoming difficulties depends on the ethical use of the concept of sustainable development; the political will of nations at a global scale; and the generation of methodologies consistent with the reality of each context. The article thus provides a framework for reflecting on the possibilities and limitations of cultural sustainability.
\end{abstract}

Keywords: culture, cultural heritage, sustainable development, UNESCO, ONU. 
La incorporación de la cultura y el patrimonio ...

Desde la aparición del informe Brundtland (1987) diversas agencias y actores culturales, especialmente la Organización de las Naciones Unidas para la Educación, la Ciencia y la Cultura (UNESCO), han trabajado arduamente por integrar a la cultura en el desarrollo sostenible. La sostenibilidad, entendida como la consecución de la armonía entre el medioambiente y el ser humano para garantizar su desarrollo y bienestar, implica que la cultura sea asumida como la base de todos los procesos humanos. Este argumento ha sido clave en el trabajo que han venido realizando los diversos organismos a fin de incorporar la dimensión cultural y por tanto el patrimonio - como pilar independiente del desarrollo sostenible. El interés por incluir claramente a la cultura en los Objetivos para el Desarro1lo (ODS) de las diferentes agendas adoptadas por la Asamblea General de las Naciones Unidas (ONU) responde a la percepción sobre su capacidad transformadora que proporcionaría ventajas sociales, económicas y medioambientales.

El siguiente artículo propone analizar como caso de estudio el proceso de incorporación de la cultura en la sostenibilidad a través de la producción documental, normativa y demás eventos relevantes que los organismos oficiales internacionales han producido. El fin último de este trabajo es entender por qué esta integración ha resultado compleja y cuáles serían las posibilidades y limitaciones para lograr la sostenibilidad cultural. Con el propósito de responder a estas cuestiones, nuestra investigación ha utilizado una metodología comparativa con el fin de contrastar las diferentes definiciones, acuerdos y normas asociadas a los conceptos de cultura, sostenibilidad y desarrollo. De este modo se pretende aportar una base histórica que nos permita realizar una reflexión bien documentada y que nos faculte a debatir críticamente sobre el papel de la sostenibilidad cultural en el desarrollo humano. Para ello, en primer lugar se examinan los debates surgidos a causa de la dificultad de definir los conceptos antes mencionados en el contexto del desarro1lo, analizando también las restricciones e implicaciones éticas que la utilización de los mismos plantea. A continuación se ofrecerá una lectura diacrónica a manera de resumen que resaltará los acontecimientos clave que se han llevado a cabo en la promoción y reclamo de la cultura y el patrimonio dentro del paradigma del desarrollo sostenible y por ende, en las políticas mundiales de desarrollo. Este apartado se divide en tres etapas: 1972 a 1986, 1987 a 2011 y desde 2012 hasta la actualidad, tomando como partida el año de la Convención de Patrimonio Mundial. 
En el último apartado se aportan una serie de propuestas acerca de la relación entre cultura, patrimonio y desarrollo sostenible que identifican las oportunidades y problemas que supone tal vínculo.

\subsection{La dificultad de definir sostenibilidad y cultura en el contexto del desarrollo}

La primera definición de desarrollo sostenible surgida a finales de los años 1980 en el llamado Informe Brundtland (1987) se realizó como reacción a la degradación del medio ambiente y el exceso de consumo en las economías altamente industrializadas (Hosagrahar, 2015). La sostenibilidad quedaba de este modo definida como "el desarrollo que satisface las necesidades del presente sin comprometer la habilitad de las futuras generaciones de garantizar sus propias necesidades" (Informe Brundtland, 1987: 27). A partir de entonces el término se ha popularizado, y ha sido asumido en otros ámbitos, lo que ha generado una amplia variedad de interpretaciones semánticas que van desde ideologías políticas (Harrison, 2013; Pezzoli, 1997; Stylianou-Lambert et al., 2015) hasta el impacto ambiental de las acciones del ser humano (Auclair, Fairclough, et al., 2015; Brocchi, 2010; Dessein et al., 2015; Hosagrahar, 2015; Rodwell, 2007; Stubbs, 2004). El concepto de desarrollo sostenible y sus diversas interpretaciones son a menudo objeto de crítica, pues implica dos discursos en conflicto (Rodwell, 2007). Por un lado, el del desarrollo económico apoyado en el capitalismo que supone el usufructo de los recursos naturales y el capital humano para la acumulación de riqueza, y por el otro, el de la sostenibilidad, que implica un equilibrio entre economía, medioambiente y sociedad. Esto supondría que la sostenibilidad está subordinada a una lógica neoliberal (Brocchi, 2010; Dylan, 2012; Holden, 2017; Thomson, 2015; Schurman, 1996; White, 2016), lo que ha provocado que el desarrollo sostenible sea considerado un oxímoron (Auclair etal, 2015; Redclif, 2005; Soini y Dessein, 2016).

La dificultad de emparejar la sostenibilidad con el desarrollo ha provocado que su interrelación sea considerada una paradoja, en cuanto propone un equilibrio que hasta ahora no se ha cumplido. Esto ha supuesto que varios autores consideren la sostenibilidad como una categoría independiente considerándole de forma cualitativa e integral, al contrario de lo que implica el desarrollo exclusivamente económico económico(De Vries y Petersen, 2009; Mišetić y Ursić, 2015; van Oers y Pereira Roders, 2012; Barbero Franco, 2011; Harrison, 2013; Rodwell, 2007, Soiniy Dessein, 2016).

\section{La cultura en el modelo de desarrollo sostenible}


La incorporación de la cultura y el patrimonio ...

Esta visión más holística, ha llevado a los diversos actores culturales a tratar de incorporar al paradigma sostenible un tercer concepto, el de cultura, lo que supone un desafío en la medida en que este también se ha visto afectado por las múltiples definiciones y categorizaciones que simultáneamente existen del mismo (1) (Soini y Dessein, 2016).

La inclusión de la cultura en el desarrollo sostenible presenta el reto de cómo entender este concepto. La tendencia actual es distinguir entre un concepto amplio de cultura como la base de todos los ámbitos del quehacer humano, y uno limitado basado solo en productos intelectuales, espirituales y estéticos (Soini y Dessein, 2016). Esta dualidad plantea desafíos adicionales a los ya existentes que hacen complicado entender a la cultura en el contexto del desarrollo sostenible. El uso de la definición amplia de cultura por muchos autores (Barbero Franco, 2011; Dessein et al., 2015; CMCD, 1997; Walker et al., 2014; Wu, Fan, y Chen, 2016) implica el riesgo de considerar que todo lo que produce la sociedad es cultura, lo que claramente no es cierto. Esto es indiscutible en el caso de la contaminación medioambiental por citar un ejemplo, pues a pesar de ser resultado de la acción humana no se podría de ninguna manera considerar como cultura. Por último queremos apuntar que si bien los términos cultura y sostenibilidad están interrelacionados, pues el primero es fundamental para el segundo, lo que dificulta el análisis de la relación entre ambos es que "son conceptos sujetos a procesos sociales, políticos de negociación científica, interpretación, y adaptación que escapan a cualquier definición final” (Soini y Dessein, 2016:3).

Las dificultades de definir sostenibilidad y cultura en el contexto del desarrollo que hemos expuesto en este apartado nos lleva a concluir que existe una responsabilidad ética en su uso. Esta hace necesario que en cada situación sea imperativo aclarar el significado semántico que se le está dando a cada uno de estos términos, dada la ambigüedad que puede descontextualizar su sentido aprovechando su uso con fines menos altruistas.

\subsection{La sostenibilidad cultural}

El término sostenibilidad cultural se menciona por primera vez en 1995 en el marco del informe de la Comisión Mundial para la Cultura y el Desarrollo denominado Nuestra Diversidad Creativa, asociándolo así al acceso a los recursos culturales (CMCD,1997).El concepto, sin embargo, no lo incluirá la UNESCO hasta 2001 
en la Declaración Universal sobre la Diversidad Cultural con la intención de justificar la dimensión cultural como el cuarto pilar del modelo sostenible (Walker et al., 2014). Condensando lo que diversos autores afirman sobre la sostenibilidad cultural, podríamos decir que es la manifestación y práctica de la cultura tradicional y nueva como resultado de un constante proceso creativo y de libre expresión individual y colectiva que enlaza pasado y presente en el espacio físico artificial y natural generando una dinámica que caracteriza la solución de problemas, las formas de vida, el arte, el conocimiento, el patrimonio tangible e intangible y especialmente la identidad que forman en conjunto un legado que se espera llegue al futuro (Auclair et al., 2015; Barbero Franco, 2011; Galla, 2012; Grimwade y Carter, 2000; Latour, 2007; Mišetić y Ursić, 2015; Soini y Birkeland, 2014; Tweed y Sutherland, 2007).

El desarrollo sostenible implica un compromiso con el porvenir del ser humano y el planeta que le da sentido, pero comporta un riesgo pues, como apunta Reid (2013), la sostenibilidad plantea un impreciso idealismo no chequeado en la realidad ya que aún no tenemos certeza de las necesidades y escenarios ecológicos o económicos del futuro y esto cuestiona su efectiva realización. Por otro lado, es necesario apuntar que si el "desarrollo sostenible es aquel que genera sus propias condiciones para producirse y mantenerse" (Barbero Franco, 2011:77), queda la duda de si tales condiciones pueden llegar a no afectar a la sostenibilidad cultural.

El Marco Conceptual para la Cultura y Sostenibilidad propuesto por Soini y Dessein (2016:3) plantea que, en realidad, la sostenibilidad cultural es posible porque existe una relación inherente de la cultura con la economía, la sociedad y el medioambiente. Estos autores mantienen que el concepto de sostenibilidad cultural se basa en tres representaciones que la cultura tiene dentro del paradigma sostenible. La primera es la consideración de esta como pilar independiente paralelo a los pilares medioambiental, económico y social. La denominan cultura en la sostenibilidad, porque reconoce la importancia de la preservación de lo que, siguiendo a Bourdieu (2011), denominan capital cultural (2). Esta primera representación asume que la cultura es un recurso que debe ser preservado y distribuido de forma equitativa inter e intra-generacionalmente.

La cultura para la sostenibilidad es la segunda forma que Soini y Dessein (2016) proponen para entender la cultura dentro del paradigma sostenible Se refirieren a esta como una fuerza mediadora que permite conceptualizar, regular y dar 
La incorporación de la cultura y el patrimonio ...

forma a los procesos del desarrollo a través del valor de la cultura material e inmaterial, y los criterios y percepciones culturales de los diversos actores en la consecución de la sostenibilidad. En definitiva, entienden la cultura como un recurso de contextualización, mediación y equilibrio entre todos los pilares del modelo que permite la sostenibilidad.

Por último, la tercera representación que nos proponen Soini y Dessein (2016) es la cultura como sostenibilidad, en la que se plantea que el desarrollo es en sí un proceso cultural que convierte la cultura en una dimensión global de la sostenibilidad. Al integrar el conjunto de dimensiones del modelo, este enmarca la variedad de creencias, ideas e interpretaciones que poseen los diversos actores sobre la realidad. En esta tercera representación no es por lo tanto posible separar la cultura de la formulación de políticas ni de todas las demás interacciones necesarias para garantizar la consecución de los Objetivos para el Desarrollo de los que hemos hablado más arriba.

La cultura, de acuerdo con el marco conceptual de Soini y Dessein (2015) posee dos valores interrelacionados, uno intrínseco y otro instrumental. El primero se refiere al conjunto de valores que se relacionan con la experiencia subjetiva de la cultura a nivel intelectual, emocional y espiritual que se reflejan en la estética, los sitios históricos, el patrimonio, el arte y el conocimiento científico. El segundo, el valor instrumental, se considera un medio para generar impactos sociales, económicos o medioambientales que garantizan el bienestar humano a través de la investigación, la educación, la valoración, la creación, la acumulación y la difusión de conocimientos y experiencias de la naturaleza, es decir, cualquier proceso cultural que facilite los procesos de desarrollo. Ambos valores garantizan el funcionamiento y evolución de la sociedad, la economía y el medioambiente hacia la sostenibilidad, porque la cultura es un motor de cambio que opera de manera transversal a todas las dimensiones.

\subsection{El modelo sostenible y el problema de la incorporación de la cultura y el patrimonio}

Para entender las dificultades que la incorporación de la cultura (y, como parte de ella, del patrimonio) ha tenido en la sostenibilidad es necesario realizar una lectura diacrónica de las diferentes medidas que se han ido tomando en estos últimos cuarenta y cinco años, partiendo de 1972.

\section{Sostenibilidad y cultura: una difícil inserción}


En este año la Convención para la Salvaguarda del Patrimonio Mundial Cultural y Natural de la UNESCO marca el inicio de un cambio global en la manera de preservar los bienes patrimoniales. Esto se debe a que representa el primer instrumento clave a nivel internacional que establece la conexión entre cultura y naturaleza, proponiendo que la conservación y la valoración del patrimonio influye decisivamente en la mejora de la calidad de vida de las comunidades, asegurando el legado a las futuras generaciones (UNESCO, 1972: Art. 4-5).

La Convención de 1972 genera una serie de conferencias a nivel mundial que tendrán como fin alinear los nuevos preceptos relacionados con el concepto de patrimonio común de la humanidad con los objetivos políticos, económicos y sociales de las naciones (Cuadro 1). Es necesario apuntar que la Convención, si bien reconoce la relación del ser humano con el medioambiente, surge divida entre la consideración de lo natural y lo cultural y de lo material e inmaterial, una falta de integridad que llevará muy pronto a problemas (Harrison, 2013). Consciente de esto la UNESCO crea ya en 1976 la Recomendación relativa a la Participación y la Contribución de las Masas Populares en la Vida Cultural (UNESCO, 1976). Según esta la eliminación de las desigualdades económicas y sociales dependería de la afirmación de la autenticidad, la dignidad y la identidad cultural. Estos tres factores permitirían el desarrollo del turismo y de las industrias culturales a través de políticas socioeconómicas adecuadas y respetuosas del medio ambiente. Con estos argumentos la UNESCO pretende demostrar que el patrimonio puede ser fuente de beneficio económico, social y ambiental lo que, traducido en términos actuales, significaría incluir la noción de sostenibilidad en los modelos de gestión patrimonial.

El desarrollo sostenible como concepto se torna popular a partir de la publicación por parte de la Comisión Mundial sobre el Medioambiente y el Desarrollo de la ONU del Informe Brundtland (1987), que promueve una visión global de responsabilidad en la gestión del medioambiente para alcanzar el desarrollo. Sin embargo, no será sino hasta la Conferencia de las Naciones Unidas sobre el Medio Ambiente y el Desarrollo de 1992 (también conocida como Conferencia de Río y Cumbre de la Tierra) cuando las cuestiones relativas a la sociedad, la justicia, la participación local, el acceso a la educación, la identidad y el sentido de pertenencia pasan a formar parte desarrollo sostenible. La Conferencia de Río daría como resultado la Declaración de Río y denominado Programa 21 para el Desesarrollo. 
La incorporación de la cultura y el patrimonio ...

Estos extrapolan el concepto de desarrollo sostenible a un modelo que se apoya en tres pilares: el medioambiente, la economía y la sociedad. Es decir, se relega el ámbito cultural a desarrollarse de manera independiente (Figura 1) a pesar de que solo cinco años antes el tema de la cultura y el desarrollo se había tratado ampliamente y de forma conjunta en reuniones como la Conferencia Mundial sobre Las Políticas Culturales (MONDIACULT) de 1982 (UNESCO, 1982) (Cuadro 1).

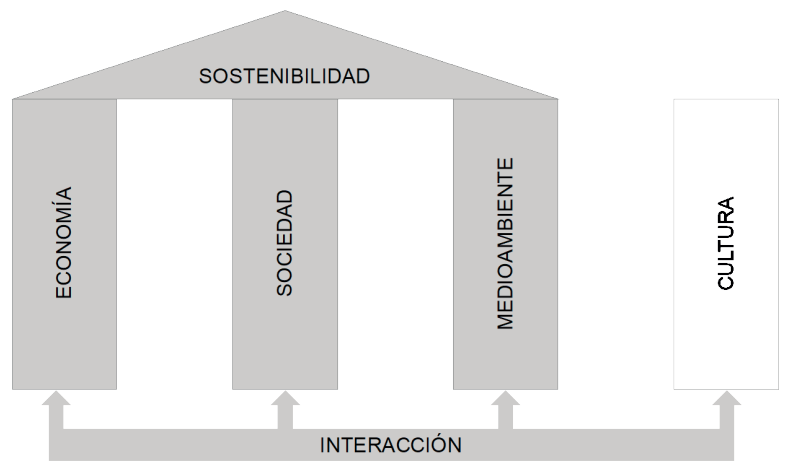

Figura1. El Modelo Sostenible (elaboración propia).

\subsection{La cultura como pilar independiente de la sostenibilidad: 1987-2011.}

El Informe Brundtland (1987) marca el inicio del desarrollo del modelo sostenible, y a partir de este documento, existen dos momentos de relevancia en el proceso de reclamo para la incorporación de la dimensión cultural en el desarrollo sostenible. El primero se produce entre 1988 y 2000, iniciándose con la Declaración del Decenio Mundial para el Desarrollo Cultural (1988-1997). Este propone políticas y actividades para el desarrollo humano y el enriquecimiento de las identidades culturales en relación con las otras dimensiones del desarrollo (UNESCO,1990). Sin embargo, los esfuerzos realizados en esta declaración por mostrar la importancia de la cultura en el desarrollo no se reflejan en la Declaración de Río Sobre Medio Ambiente y Desarrollo de 1992. Este fracaso vendrá a demostrar que el concepto de desarrollo sostenible, tal y como entonces se estaba definiendo, se enfocaba casi exclusivamente a lo económico, ecológico y social, siendo escasas las menciones referentes a temáticas culturales y realizándose estas siempre bajo el paraguas de lo social (Auclair et al., 2015).

Como consecuencia de Río la UNESCO, en su búsqueda por el reconocimiento de la cultura en el paradigma sostenible, produce una serie de documentos y confe- 
conferencias que justifican la toma de una serie de nuevas decisiones a lo largo de la década de 1990 (Vlassis, 2015). La más relevante es la adopción de la categoría de Paisaje Cultural en 1992 que se incorpora a los criterios de la Convención de Patrimonio Mundial. Gracias a esta en 1999 fue posible introducir el concepto de sostenibilidad aplicado al patrimonio dentro de las directrices prácticas para la implementación de la Convención, convirtiéndose así estas en el primer instrumento internacional en reconocer la utilización sostenible de los paisajes culturales con la finalidad de mejorar la calidad de vida de las comunidades (CPM, 2003)

De vuelta a 1992, a la nueva y más integral visión de paisaje cultural incorporada en aquel año seguiría la propuesta de Nuestra Diversidad Creativa (UNESCO, 1997). Esta establece que la cultura no es un medio, sino la base de todos los procesos que incumben al desarrollo. Este argumento es un año más tarde ratificado en la declaración realizada en el llamado Documento de Estocolmo que se refiere a la cultura como "una fuerza económica y política, y un factor de poder en sí mismo" (ICOMOS, 1998. Punto 7: 9). Los esfuerzos de la UNESCO por acercarse al paradigma sostenible durante la última década del milenio terminan con la conferencia La cultura cuenta de 1999 producida con el apoyo del Banco Interamericano de Desarrollo, y en esta se justifica el vínculo entre cultura y desarrollo (Wolfensohn, 2000). Sin embargo, como explicaremos a continuación, el intento por parte de la UNESCO no obtendrá el resultado esperado.

El segundo momento de relevancia en el proceso de reclamo para la incorporación de la dimensión cultural en el desarrollo sostenible comienza en el año 2000 con la Declaración del Milenio de la ONU y sus denominados Objetivos del Milenio (ONU, 2000) de los que nuevamente la cultura queda excluida. Esta situación hace que ciertos sectores decidan organizarse para promover un esfuerzo internacional que busque las bases teóricas y las herramientas técnicas que permitan crear programas en torno a la cultura como aspecto inherente al desarrollo sostenible. Esto se logra enfatizando la diversidad, los derechos de las minorías, la producción de marcos legales y políticas de protección y preservación del patrimonio como forma de respeto y participación comunitaria en el contexto de las dinámicas urbanas (Cuadro 1). Dentro de dicha producción son importantes la Declaración de la ONU del Año para el Patrimonio Mundial de 2002, la Convención para la Salvaguardia del Patrimonio Cultural Inmaterial en 2003, la Convención sobre la Protección y la Promoción de la Diversidad de las Expresiones 
La incorporación de la cultura y el patrimonio ...

Culturales de 2005. También lo fueron la adopción de la Agenda 21 del Programa 21 por parte de la organización Ciudades y Gobiernos Locales Unidos (UCLG) en 2010, que será la primera medida que resalta el papel de la cultura en las políticas urbanas como el cuarto pilar del desarrollo sostenible (Soini y Birkeland, 2014). Finalmente, la Recomendación sobre el Paisaje Urbano Histórico (UNESCO, 2011) propone una visión integral del patrimonio en el contexto de la sostenibilidad.

\subsection{Desde 2012 hasta nuestros días}

Con motivo del 40 aniversario de la Convención en el año 2012 se desarrolla la conferencia Patrimonio Mundial y Desarrollo Sostenible: el Papel de las Comunidades Locales (UNESCO, 2015). Esta reunión refuerza, a través de la denominada Visión de Kyoto (UNESCOa, 2012), las posiciones adoptadas en décadas anteriores por la UNESCO, sus órganos consultivos y varias otras organizaciones internacionales involucradas en la tutela de los bienes culturales (Bandarin, 2015). El documento de Kyoto hace referencia a la inclusión de personas, políticas y patrimonio en la sostenibilidad como condición para asegurar la conservación a largo plazo y la preservación del Valor Universal Excepcional de los sitios Patrimonio Mundial.

La Visión de Kyoto, junto con otros varios documentos y conferencias (Cuadro 1), especialmente Río +20 El futuro que queremos (2012), crean un nuevo clima que lleva a la cultura a la arena política de la Agenda Post 2015 (la agenda que ha venido a sustituir al documento de los Objetivos del Milenio) y de la Agenda 2030 de 2015 (actualización de la Agenda Post 2015). En ellas, a pesar de que se reconoce de manera muy general el papel de la cultura, en la práctica se la sigue excluyendo de los procesos de planificación y las políticas de desarrollo (Figura 1).

La oposición a integrar la cultura como dimensión por sí sola ha llevado a problemas producto de las ambiguas e imprecisas definiciones de los términos de las que ya hemos hablado anteriormente en este artículo, que dificulta su entendimiento sobre el alcance y límites de su uso al momento de intentar desarrollar metodologías por parte de los que gestionan la cultura. Otros problemas se derivan del factor intergubernanmental que, de acuerdo con Antonios Vlassis (2015), es responsable de que la cultura no se ha incorporado al paradigma del desarrollo sostenible. Según apunta este autor, la negativa de la mayoría de los gobiernos europeos y norteamericanos a esta integración ha llevado a que las asambleas de la ONU 
donde se ha tratado este aspecto hayan estado marcadas por la ausencia de representantes de estos países. Su aparente falta de interés en el tema reflejada en su no comparecencia a estas reuniones es relevante dado su papel de principales contribuyentes a la ayuda al desarrollo internacional (Vlassis, 2015: 1656). En Nueva York, asegura Vlassis (2015), existe una doble realidad de la Asamblea General por la que, aunque teóricamente todas las delegaciones están a favor de la inclusión de la cultura, en la práctica se muestran indiferentes cuando se trata de concretar cómo implementarla.

Esta reticencia por parte de los gobiernos ha estado compensada, sin embargo, por los esfuerzos de instituciones como la UNESCO, que en su afán por conseguir el reconocimiento de la cultura como pilar independiente de la sostenibilidad, ha realizado un esfuerzo tanto en la producción científica (convenios, reuniones, recomendaciones) como técnica (guías para la implementación de la Convención, nuevas directrices, libros editados, manuales, etc.) en torno al tema de la inclusión de la sostenibilidad en la gestión de los Sitios Patrimonio Mundial (Albert, 2015; Auclair et al., 2015; Barthel-Bouchier, 2016; Galla, 2012; Hassan y Lee, 2015; Labadi, 2017; Rodwell, 2007). Estos enfoques han actualizado el concepto de patrimonio vinculándolo estrechamente con el desarrollo en un momento en el que, como hemos visto, la sostenibilidad es un aspecto clave dentro de las agendas de planificación a nivel mundial.

Los argumentos que la UNESCO ha generado para introducir la cultura en el modelo de desarrollo sostenible han puesto de manifiesto la importancia de reconocer la capacidad creativa y de adaptación del ser humano. Esto ha supuesto en el contexto de esta institución que con el fin de dar sentido a los conceptos para el desarrollo de metodologías, han de generarse normativas (Soini y Dessein, 2016), que son justamente las que resumimos en el cuadro a continuación. 
La incorporación de la cultura y el patrimonio ...

Cuadro 1.

Producción global relevante en el marco de la cultura y sostenibilidad

\begin{tabular}{|c|c|c|}
\hline Período & Evento & Institución \\
\hline \multirow{4}{*}{1972} & $\begin{array}{l}\text { Declaración de Estocolmo sobre el } \\
\text { Medio Humano. }\end{array}$ & ONU \\
\hline & $\begin{array}{l}\text { Recomendación sobre la Protección } \\
\text { en el Ámbito Nacional del Patrimonio } \\
\text { Cultural Natural. }\end{array}$ & UNESCO \\
\hline & $\begin{array}{l}\text { Convención para la Salvaguarda del } \\
\text { Patrimonio Mundial Cultural y Natu- } \\
\text { ral. }\end{array}$ & UNESCO \\
\hline & $\begin{array}{l}\text { Creación del Programa del Medio Am- } \\
\text { biente de las Naciones Unidas }\end{array}$ & PNUMA \\
\hline \multirow[b]{2}{*}{1975} & $\begin{array}{l}\text { Carta de Belgrado para la Educación } \\
\text { Ambiental. }\end{array}$ & UNESCO- PNUMA \\
\hline & $\begin{array}{l}\text { Creación de la Organización Mundial } \\
\text { del Turismo. }\end{array}$ & OMT \\
\hline \multirow{3}{*}{1976} & $\begin{array}{l}\text { Recomendación de Nairobi relativa a } \\
\text { la Salvaguardia de los Conjuntos His- } \\
\text { tóricos o Tradicionales y su Función en } \\
\text { la Vida Contemporánea. }\end{array}$ & UNESCO \\
\hline & $\begin{array}{l}\text { Recomendación relativa a la Partici- } \\
\text { pación y la Contribución de las Masas } \\
\text { Populares en la Vida Cultural }\end{array}$ & UNESCO \\
\hline & Conferencia Hábitat I. & ONU \\
\hline \multirow[b]{2}{*}{1978} & $\begin{array}{l}\text { Conferencia Intergubernamental sobre } \\
\text { las Políticas Culturales en América } \\
\text { Latina y el Caribe. }\end{array}$ & UNESCO \\
\hline & $\begin{array}{l}\text { Creación del Programa de Naciones } \\
\text { Unidas para los Asentamientos Huma- } \\
\text { nos (ONU- HABITAT). }\end{array}$ & UNESCO \\
\hline 1981 & $\begin{array}{l}\text { Carta de Florencia para la Salvaguarda } \\
\text { de los Jardines Históricos. }\end{array}$ & ICOMOS \\
\hline 1982 & $\begin{array}{l}\text { Conferencia Mundial y Declaración de } \\
\text { México sobre Las Políticas Culturales } \\
\text { (MONDIACULT). }\end{array}$ & UNESCO \\
\hline 1986 & $\begin{array}{l}\text { Declaración sobre el Derecho al Desa- } \\
\text { rrollo. }\end{array}$ & ONU \\
\hline \multirow[b]{2}{*}{1987} & $\begin{array}{l}\text { Informe Brundtland Nuestro Futuro } \\
\text { Común. }\end{array}$ & $\mathrm{ONU}$ \\
\hline & $\begin{array}{l}\text { Carta de Washington para la Conser- } \\
\text { vación de Ciudades Históricas y Áreas } \\
\text { Urbanas Históricas. }\end{array}$ & ICOMOS \\
\hline
\end{tabular}

Vol. 8 (1), 2018 / EISSN: 2215 - 393462 


\begin{tabular}{|c|c|c|}
\hline 1988 & $\begin{array}{l}\text { Decenio Mundial para el Desarrollo } \\
\text { Cultural (1988-1997). }\end{array}$ & ONU \\
\hline 1989 & $\begin{array}{l}\text { Creación del Programa de las Naciones } \\
\text { Unidas para el Desarrollo. }\end{array}$ & PNUD \\
\hline 1991 & $\begin{array}{l}\text { Creación de la Comisión Mundial so- } \\
\text { bre la Cultura y el Desarrollo Indepen- } \\
\text { diente (CMCD). }\end{array}$ & ONU-UNESCO \\
\hline \multirow{4}{*}{1992} & $\begin{array}{l}\text { Conferencia de las Naciones Unidas } \\
\text { sobre el Medioambiente y el Desarro- } \\
\text { llo. (CNUMAD) }\end{array}$ & ONU \\
\hline & $\begin{array}{l}\text { Declaración de Río sobre Medioam- } \\
\text { biente y Desarrollo. }\end{array}$ & ONU \\
\hline & $\begin{array}{l}\text { Plan Mundial para el Desarrollo Soste- } \\
\text { nible. Programa y Agenda } 21\end{array}$ & ONU \\
\hline & $\begin{array}{l}\text { Introducción de la categoría de Paisaje } \\
\text { Cultural en la Convención de Patrimo- } \\
\text { nio Mundial. }\end{array}$ & UNESCO \\
\hline 1994 & $\begin{array}{l}\text { Documento de Nara sobre la Autentici- } \\
\text { dad del Patrimonio Cultural. }\end{array}$ & ICOMOS \\
\hline \multirow{3}{*}{1996} & $\begin{array}{l}\text { Asamblea General sobre el Desarro- } \\
\text { llo de los Recursos Humanos para el } \\
\text { Desarrollo }\end{array}$ & $\mathrm{ONU}$ \\
\hline & $\begin{array}{l}\text { Informe Mundial de Cultura y Desa- } \\
\text { rrollo. Nuestra Diversidad Creativa. }\end{array}$ & UNESCO \\
\hline & Hábitat II. & \\
\hline \multirow{3}{*}{1998} & $\begin{array}{l}\text { Conferencia Intergubernamental sobre } \\
\text { Políticas Culturales para el Desarro- } \\
\text { llo. Documento de Estocolmo. Plan } \\
\text { de Acción sobre las Políticas para el } \\
\text { Desarrollo. }\end{array}$ & UNESCO \\
\hline & $\begin{array}{l}\text { Creación de la Red Internacional de } \\
\text { Políticas Culturales. }\end{array}$ & UNESCO \\
\hline & $\begin{array}{l}\text { Carta de Burra para Sitios de Significa- } \\
\text { ción Cultural. }\end{array}$ & ICOMOS \\
\hline \multirow{2}{*}{1999} & $\begin{array}{l}\text { Foro del Banco Interamericano de De- } \\
\text { sarrollo sobre Cultura y Desarrollo. }\end{array}$ & BID \\
\hline & $\begin{array}{l}\text { Conferencia Internacional La Cultura } \\
\text { Cuenta. }\end{array}$ & Banco Mundial \\
\hline
\end{tabular}


La incorporación de la cultura y el patrimonio ...

\begin{tabular}{|c|c|c|}
\hline 2000 & $\begin{array}{l}\text { Declaración del Milenio de las Nacio- } \\
\text { nes Unidas. Carta de la Tierra (Objeti- } \\
\text { vos del Milenio). }\end{array}$ & $\mathrm{ONU}$ \\
\hline 2001 & $\begin{array}{l}\text { Declaración Universal sobre la Diver- } \\
\text { sidad Cultural. }\end{array}$ & UNESCO \\
\hline \multirow{4}{*}{2002} & $\begin{array}{l}\text { Cumbre Mundial sobre el Desarrollo } \\
\text { Sostenible (CMDS). }\end{array}$ & ONU \\
\hline & $\begin{array}{l}\text { Año de las Naciones Unidas para el } \\
\text { Patrimonio Cultural Mundial. }\end{array}$ & ONU \\
\hline & $\begin{array}{l}\text { Declaración de Budapest sobre Patri- } \\
\text { monio Mundial. }\end{array}$ & UNESCO \\
\hline & $\begin{array}{l}\text { Declaración de Johannesburgo sobre } \\
\text { Desarrollo Sostenible y Plan de Apli- } \\
\text { cación de Johannesburgo. }\end{array}$ & ONU \\
\hline 2003 & $\begin{array}{l}\text { Convención para la Salvaguardia del } \\
\text { Patrimonio Cultural Inmaterial. }\end{array}$ & UNESCO \\
\hline \multirow{2}{*}{2004} & $\begin{array}{l}\text { Informe sobre Desarrollo Humano. La } \\
\text { Libertad Cultural en el Mundo Diver- } \\
\text { so de Hoy }\end{array}$ & PNUD \\
\hline & $\begin{array}{l}\text { Creación de Organización Mundial de } \\
\text { Ciudades y Gobiernos Locales Unidos. }\end{array}$ & CGLU \\
\hline \multirow{4}{*}{2005} & $\begin{array}{l}\text { Convención sobre la Protección y Pro- } \\
\text { moción de la Diversidad de las Expre- } \\
\text { siones Culturales. }\end{array}$ & UNESCO \\
\hline & $\begin{array}{l}\text { Declaración de Xi'an sobre la Conser- } \\
\text { vación del Entorno de las Estructuras, } \\
\text { Sitios y Áreas Patrimoniales. }\end{array}$ & ICOMOS \\
\hline & $\begin{array}{l}\text { Protocolo de Kioto sobre Cambio } \\
\text { Climático. }\end{array}$ & ONU \\
\hline & $\begin{array}{l}\text { Memorándum de Viena sobre Patri- } \\
\text { monio Cultural y arquitectura contem- } \\
\text { poránea-Gestión del Paisaje Urbano } \\
\text { Histórico. }\end{array}$ & UNESCO \\
\hline 2006 & $\begin{array}{l}\text { Carta Cultural Iberoamericana de la } \\
\text { Organización de Estados Iberoameri- } \\
\text { canos. }\end{array}$ & OEI \\
\hline
\end{tabular}




\begin{tabular}{|c|c|c|}
\hline \multirow{3}{*}{2007} & $\begin{array}{l}\text { Incorporación de las Comunidades } \\
\text { para fortalecer la credibilidad de la } \\
\text { Lista de Patrimonio en la } 31^{\text {a }} \text { Reunión } \\
\text { del Comité de Patrimonio Mundial. }\end{array}$ & UNESCO \\
\hline & $\begin{array}{l}\text { Declaración de las Naciones Unidas } \\
\text { sobre los Derechos Indígenas. }\end{array}$ & ONU \\
\hline & $\begin{array}{l}\text { Conferencia de Bali sobre Cambio } \\
\text { Climático. }\end{array}$ & ONU \\
\hline \multirow{2}{*}{2008} & $\begin{array}{l}\text { Declaración de Quebec sobre la Pre- } \\
\text { servación del Espíritu del Lugar. }\end{array}$ & ICOMOS \\
\hline & $\begin{array}{l}\text { Carta Ename sobre Interpretación y } \\
\text { Presentación de Sitios de Patrimonio } \\
\text { Cultural. }\end{array}$ & ICOMOS \\
\hline $\begin{array}{l}2009- \\
2013\end{array}$ & $\begin{array}{l}\text { El programa de los Indicadores de } \\
\text { Cultura para el Desarrollo (IUCD). }\end{array}$ & UNESCO \\
\hline \multirow{2}{*}{2010} & $\begin{array}{l}\text { Resolución 65/166 sobre Cultura y } \\
\text { Desarrollo. }\end{array}$ & ONU \\
\hline & $\begin{array}{l}\text { Reunión de Paraty sobre Patrimonio } \\
\text { Mundial y Desarrollo Sostenible. }\end{array}$ & UNESCO \\
\hline \multirow{4}{*}{2011} & $\begin{array}{l}\text { Declaración de París sobre el Patrimo- } \\
\text { nio como Motor del Desarrollo }\end{array}$ & ICOMOS \\
\hline & $\begin{array}{l}\text { Resolución } 66 / 208 \text { sobre Cultura y } \\
\text { Desarrollo }\end{array}$ & ONU \\
\hline & $\begin{array}{l}\text { Plan estratégico de acción para la } \\
\text { implementación de la Convención de } \\
\text { Patrimonio Mundial 2012-2022. }\end{array}$ & UNESCO \\
\hline & $\begin{array}{l}\text { Recomendación sobre el Paisaje Urba- } \\
\text { no Histórico (PUH). }\end{array}$ & UNESCO \\
\hline \multirow{2}{*}{2012} & $\begin{array}{l}\text { Conferencia sobre el Desarrollo Sos- } \\
\text { tenible Río+20 El Futuro que Quere- } \\
\text { mos. }\end{array}$ & ONU \\
\hline & $\begin{array}{l}\text { Publicación de la Agenda de Desarro- } \\
\text { llo Post } 2015 .\end{array}$ & ONU \\
\hline
\end{tabular}


La incorporación de la cultura y el patrimonio ...

\begin{tabular}{|c|c|c|}
\hline \multirow{4}{*}{2012} & $\begin{array}{l}\text { Aniversario } 40 \text { de la Convención de } \\
\text { Patrimonio Mundial. Patrimonio } \\
\text { Mundial y Desarrollo Sostenible: el } \\
\text { papel de las comunidades locales. }\end{array}$ & UNESCO \\
\hline & $\begin{array}{l}\text { Reunión de expertos en Ouro Preto } \\
\text { sobre Patrimonio y Desarrollo Soste- } \\
\text { nible. }\end{array}$ & UNESCO \\
\hline & $\begin{array}{l}\text { Propuesta de Toyama para el Desa- } \\
\text { rrollo Sostenible. }\end{array}$ & UNESCO \\
\hline & $\begin{array}{l}\text { Programa de Patrimonio Mundial y } \\
\text { Turismo Sostenible. }\end{array}$ & UNESCO- OMT \\
\hline \multirow{6}{*}{2013} & $\begin{array}{l}\text { Declaración de Hangzhou Cultura: } \\
\text { Clave del Desarrollo Sostenible. }\end{array}$ & UNESCO \\
\hline & $\begin{array}{l}\text { Resolución 68/223 sobre Cultura y } \\
\text { Desarrollo. }\end{array}$ & ONU \\
\hline & $\begin{array}{l}\text { Examen Ministerial Anual del Conse- } \\
\text { jo Económico y Social (ECOSOC) de } \\
\text { las Naciones Unidas sobre Cultura y } \\
\text { Desarrollo. }\end{array}$ & ONU \\
\hline & $\begin{array}{l}\text { Foro Mundial de la Cultura en el } \\
\text { Desarrollo. Promesa de Bali. }\end{array}$ & UNESCO \\
\hline & $\begin{array}{l}\text { Tercer Informe sobre la Economía } \\
\text { Creativa } 2013 .\end{array}$ & ONU \\
\hline & $\begin{array}{l}\text { Federación Internacional de Conse- } \\
\text { jos de Artes y Agencias Culturales: } \\
\text { Cultura como Objetivo en la Agenda } \\
\text { para el Desarrollo Post-2015. }\end{array}$ & FICAAC \\
\hline 2014 & $\begin{array}{l}\text { XVIII Asamblea General del } \\
\text { ICOMOS Patrimonio y paisajes } \\
\text { culturales como valores humanos. }\end{array}$ & ICOMOS \\
\hline
\end{tabular}

Vol. 8 (1), 2018 / EISSN: 2215 - 393466 


\begin{tabular}{|c|l|c|}
\hline \multirow{5}{*}{2014} & $\begin{array}{l}\text { Tercer Foro Mundial sobre la Cultura } \\
\text { e Industrias Culturales Declaración de } \\
\text { Florencia Sobre Cultura, Creatividad y } \\
\text { Desarrollo Sostenible. Investigación, } \\
\text { Innovación, Oportunidades. }\end{array}$ & UNESCO \\
\hline \multirow{5}{*}{2015} & $\begin{array}{l}\text { Resolución 70/214 sobre Cultura y De- } \\
\text { sarrollo }\end{array}$ & $\begin{array}{l}\text { Publicación de la Agenda 2030 para el } \\
\text { Desarrollo Sostenible. }\end{array}$ \\
\cline { 2 - 4 } & $\begin{array}{l}\text { Acuerdo de París sobre el Cambio Cli- } \\
\text { mático. }\end{array}$ & ONU \\
\hline 2016 & Hábitat III. & ONU \\
\hline
\end{tabular}

\section{Posibilidades, problemas y limitaciones de la sostenibilidad cultural para el patrimonio}

Tras haber repasado las dificultades que se han encontrado en el intento de buscar un reconocimiento al potencial aporte de la cultura en la sostenibilidad, veremos qué posibilidades y limitaciones ofrecen los bienes patrimoniales y especialmente los Sitios Patrimonio Mundial en la consecución de la sostenibilidad. Esto es útil porque los bienes y sitios patrimoniales son recursos finitos que garantizan la satisfacción de necesidades a largo plazo como la identidad, el sentido de pertenencia y otras que se explican a continuación. De este modo, el argumento del patrimonio refuerza las razones que justifican la incorporación de la cultura en la sostenibilidad, y al hacerlo, obliga a los entes encargados de su tutela a replantear la manera de gestionarlo.

\subsection{Las posibilidades}

Para analizar las posibilidades de la incorporación de la cultura - y por tanto del patrimonio - a la sostenibilidad examinaremos cuáles son sus posibilidades en relación con lo social, lo económico y lo medioambiental. En referencia al aspecto social, la sostenibilidad cultural a través del patrimonio permite a un grupo de ac- 
La incorporación de la cultura y el patrimonio ...

actores ejercer sus derechos culturales por medio de la redefinición de los bienes patrimoniales a través de la asimilación de diversas visiones. Esto implica un esfuerzo cooperativo entre el conocimiento especializado y el comunitario que conlleva a la posibilidad de desempeñar una tutela y gobernanza del mismo que permita preservarlo y transmitirlo, sin provocar rupturas sociales relativas a clase socioeconómica o etnia (García Canclini y Criado, 1999). La gestión participativa de la herencia patrimonial es capaz de satisfacer una variedad de aspiraciones y necesidades artísticas, estéticas, cognitivas e incluso de recreación. La carga histórica y simbólica de su discurso mediante la memoria y el aporte colectivo sirve de marco a fin de mejorar la calidad de vida, el bienestar y los valores culturales de las comunidades en el territorio. En el ámbito sociopolítico, el patrimonio también tiene un valor en el contexto nacional afirmando una identidad que contribuye al sentido de solidaridad entre sus ciudadanos, lo que tiene implicaciones políticas relacionadas con el ejercicio y compromiso desde el sector cívico, las organizaciones públicas y privadas para dar significado a lo que entendemos por identidad cultural nacional.

En términos económicos los bienes patrimoniales son fuente de ingresos a través del aprovechamiento turístico de los recursos culturales materiales que favorece a una amplia red afín a estos (hoteles, restaurantes, comercios, etc), y de recursos como la música popular, la artesanía, la práctica de oficios tradicionales y la ocupación de los profesionales de la cultura, por citar algunos ejemplos. La efectiva administración de los recursos patrimoniales promueve la reutilización económica de los espacios que diversifica las actividades lo que supone un aumento de la calidad de vida. En definitiva posibilita el desarrollo económico, la generación de empleo y las posibilidades de inversión.

Desde el punto de vista medioambiental, la sostenibilidad cultural a través del patrimonio rescata el uso de aquellos saberes tradicionales cuya práctica se ha considerado respetuosa con la ecología. También posibilita la reutilización de las infraestructuras históricas para vivienda y servicios como una manera de mitigar el uso de recursos finitos e incluso la contaminación. De este modo permite entender cómo el ser humano se ha relacionado con su medioambiente natural y construido facilitando el acceso al conocimiento acumulado sobre las tecnologías y resolución de problemas en el territorio con las implicaciones que esto tiene en su cultura y por lo tanto en su forma de vida. 
Por último es necesario resaltar la importancia de la herencia patrimonial para la cultura como tal, pues como sistema de valores es una fuente de significado que vincula a las personas con los objetos y productos culturales. De este modo, no solo preserva los testimonios culturales anteriores sino que además desarrolla los actuales mediante la creatividad e innovación. Es en la posibilidad de interacción del patrimonio con las formas culturales donde se manifiesta su poder de mediación, ya que, vinculado a la creación cultural, es una gran fuerza crítica y democrática y una de las mejores maneras de difundir diferentes mensajes a la sociedad. Esto posibilita la reinterpretación del pasado en el presente y la construcción de las nuevas capas patrimoniales del futuro. En resumen, permite el empoderamiento de los diversos actores a través del conocimiento y reconocimiento a su cultura, a fin de asumir otras cuestiones del desarrollo sostenible, como la inclusión social, el crecimiento económico y el equilibrio medioambiental.

La sostenibilidad cultural a través del patrimonio no solo presenta aspectos positivos sino también lleva a problemas que examinamos a continuación. En primer lugar hemos de citar que la falta de referencias claras a la cultura dentro de la Agenda 2030, donde el aporte cultural se menciona superficialmente con el propósito de reconocer la diversidad cultural (ONU, 2015. Punto 8 y 36), lleva a minimizar el potencial y alcance real de la cultura en las políticas para el desarrollo sostenible. Esto se evidencia también dentro de los Objetivos para el Desarrollo de las diferentes agendas adoptadas por la Asamblea General de las Naciones Unidas (ONU) donde se hace una referencia muy general a las posibilidades que el patrimonio ofrece al desarrollo sostenible a través de su conservación para la generación de turismo (ONU, 2015.Objetivo 4.7, 8.7, 11.4). En segundo lugar debemos apuntar como limitación de la sostenibilidad cultural a través del patrimonio la falta de metodologías, pues aunque las estrategias para la incorporación de la cultura en el paradigma son altamente altruistas, aún carecen de suficientes resultados medibles. Es decir, se necesita que las metodologías que se proponen sean efectivamente probadas en la práctica a fin de contar con evidencias explícitas sobre las implicaciones positivas de la cultura para la sostenibilidad y especialmente para la sociedad. Desde esta perspectiva es lógico que, ante la carencia de resultados, se haya prestado mayor atención a las dimensiones económica y medioambiental del desarrollo sostenible y no a la cultura o al patrimonio.

La dificultad de medir el aporte de la cultura en el desarrollo quizá está relacionada con la búsqueda de una estandarización de metodologías basadas en herramientas

\subsection{Los problemas $y$ limitaciones}


La incorporación de la cultura y el patrimonio ...

cuantitativas con el fin de evaluar de manera similar a todas dimensiones del modelo. Sin embargo, los métodos cuantitativos, que normalmente se basan en índices y porcentajes, no siempre resultan adecuados (Brugmann, 1997). Para entender esto solo deberíamos decir que si el porcentaje de visitas a un espacio patrimonial sirve para determinar la sostenibilidad, temas como el de la conservación de la Capilla Sixtina en los Museos Vaticanos no serían motivo de preocupación. Desde esta perspectiva, y a pesar de que existen estudios sobre la contribución de la cultura en el PIB especialmente por las industrias culturales, el tema es que no toda actividad cultural se mide igual. Hartmut Bossel (1999) sostiene que antes de desarrollar cualquier sistema de medición primero se habrá de identificar las cuestiones relevantes dentro de lo que él denomina el sistema humano (sistema social, desarrollo individual y gobierno).

La dificultad de medir las contribuciones culturales al modelo sostenible responde justamente a la estrecha vinculación entre cultura y sociedad, porque entender esta conexión desde un enfoque integral depende de diversas visiones. En este contexto, no existe una verdadera transversalidad con las múltiples disciplinas y actores al momento de desarrollar instrumentos, estrategias y políticas culturales para el desarrollo. Esto se evidencia principalmente en el ámbito del patrimonio, donde el aporte de los diferentes actores en la producción de metodologías para su gestión sostenible es limitado debido a la ausencia de métodos eficientes de participación social (en lo que debería ser un debate multilateral), y al vacío en relación con la diversidad de temáticas desde donde se tendría que tratar el tema.

La misma Agenda 2030 es un claro ejemplo del limitado aporte de los diferentes actores ya que desde una visión experta (que aún manifiesta un tradicionalismo propio de las disciplinas académicas) plantea una clara tendencia a entender a la cultura en el desarrollo como un privilegio de lo económico. Esto evidencia que la incorporación de la cultura en la investigación y las políticas de sostenibilidad, cambiaría el privilegio hasta ahora dado a la viabilidad económica y medioambiental, lo que demandaría una efectiva transdisciplinariedad en la investigación y la implicación real de los diversos actores en las políticas de sostenibilidad.

Los problemas y limitaciones presentados en este apartado han provocado que en el contexto de los Sitios Patrimonio Mundial la UNESCO y las diversas agencias y organismos internacionales para la cultura en la sostenibilidad se hayan visto limitados únicamente a marcar pautas con el fin de promover la cooperación internacional en temas de desarrollo. Esto ha llevado a una pérdida efectiva de eficacia en el momento de responder a la pregunta de cómo implementar la sostenibilidad cultural en los diversos contextos que es donde está el verdadero desafío. Más aún, cuando el papel de la cultura se desvanece de los Objetivos para el Desarrollo de la Agenda 2030, y por consiguiente, se pierde su vinculación directa con las políticas de desarrollo locales. 
El análisis desarrollado en este artículo sobre el proceso de incorporación de la cultura en el modelo de desarrollo sostenible ha comenzado por apuntar las dificultades a las que llevan las diversas definiciones de los conceptos de desarrollo sostenible y cultura. La ambigüedad de su semántica y los alcances y limitaciones de cada uno en el contexto de sostenibilidad y sus diferentes dimensiones a fin de lograr una práctica cultural ética han sido presentadas. A continuación se ha realizado un análisis del concepto de sostenibilidad cultural como término que enfatiza el papel de la cultura en, para, y como sostenibilidad, repasando las múltiples formas en las que la que esta aporta al desarrollo del ser humano. Hemos asumido en este trabajo la sostenibilidad cultural a manera de marco conceptual con el fin de dar sustento a la revisión de los documentos, conferencias, recomendaciones y reuniones que la UNESCO y los diversos organismos internacionales han producido con el fin de promocionar el valor de la cultura y el patrimonio en la sostenibilidad.

Con el fin de entender el proceso de reclamo de la incorporación de la cultura en la sostenibilidad, el artículo ha analizado por una parte los argumentos más relevantes utilizados en la defensa de la cultura para el desarrollo sostenible, enfatizando su papel mediador, transversal y como pilar independiente de la sostenibilidad. Por otra parte, se han examinado los obstáculos que han servido para ofrecer una posible explicación ante el constante impedimento a la cultura de contar con objetivos propios en los Objetivos para el Desarrollo de la Agenda 2030.

Se ha argumentado que las principales limitaciones para integral a la cultura en el desarrollo sostenible son por una parte la dificultad de definir los conceptos de cultura, sostenibilidad y desarrollo, y por la otra, la imposibilidad de la UNESCO de persuadir a los Estados Parte de la ONU de incluir claramente a la dimensión cultural como pilar independiente, lo que ha tenido repercusiones en de las políticas internacionales para el desarrollo. Se ha planteado el problema de la falta de resultados medibles como consecuencia de la carencia de metodologías interdisciplinarias y participativas. Adicionalmente, se ha referido que la autoridad tecnócrata, académica y en último termino las políticas tokenistas han contribuido a mantener un enfoque económico y medioambiental desatendiendo cuestiones que el paraguas cultural ofrece al modelo de la sostenibilidad. Finalmente en este trabajo se han apuntado las posibilidades y limitaciones del patrimonio en la creación de estrategias que faciliten la consecución de la sostenibilidad cultural. 
La incorporación de la cultura y el patrimonio ...

El análisis de los procesos de incorporación de la cultura y el patrimonio en la sostenibilidad realizado en este artículo muestra que la conciencia y apreciación sobre la contribución de estos han logrado algunos avances en las agendas para el desarrollo humano. El desafío actual se encuentra en cómo incorporar el modelo sostenible de manera efectiva en los diferentes contextos. De ahí que la mayor dificultad no sea realmente si la cultura llegará o no a convertirse en pilar independiente del paradigma sostenible, que desde aquí reclamamos como necesario, sino si las estrategias, políticas y métodos que puedan surgir a fin de integrar la sostenibilidad cultural en los distintos escenarios, responden a un estudio amplio y profundo del sistema sociocultural de cada realidad que permita el empoderamiento de los diversos actores. Solo así la sostenibilidad cultural tendrá un sentido real, tangible y medible más allá de lo que propone la teoría.

\section{Notas}

(1) Para revisiones más actuales del término cultura consultar en las referencias la última versión de la obra de Williams, R. (2014).

(2) El capital cultural se entiende como la acumulación de conocimientos, comportamientos y habilidades que uno puede aprovechar para demostrar su competencia cultural y, por lo tanto, su estatus social o posición en la sociedad.

\section{Referencias}

Albert, M.-T. (2015). Perceptions of Sustainability in Heritage Studies (Vol. 4): Walter de Gruyter GmbH \& Co KG.

Auclair, E., Fairclough, G., \& others. (2015). Theory and practice in heritage and sustainability: Between past and future: Routledge.

Bandarin, F. (2015). Sustainability in the World Heritage Convention: The Making of a Policy Framework Perceptions of Sustainability in Heritage Studies (Vol. 4): Walter de Gruyter GmbH \& Co KG.

Barbero Franco, A. M. (2011). La gestión del patrimonio histórico como instrumento para un desarrollo sostenible (Vol. 280): Universidad de Salamanca. 
Barthel-Bouchier, D. (2016). Cultural heritage and the challenge of sustainability: Routledge.

Baumgartner, R. J. (2009). Organizational culture and leadership: Preconditions for the development of a sustainable corporation. Sustainable Development, 17(2), 102-113.

Bennett, T., Grossberg, L., \& Morris, M. (Eds.). (2013). New keywords: A revised vocabulary of culture and society. John Wiley \& Sons

Bossel, H. (1999). Indicators for sustainable development: theory, method, applications. International Institute for Sustainable Development Winnipeg.

Bourdieu, P. (2011). The forms of capital.(1986). Cultural theory: An anthology, $1,81-93$.

Brocchi, D. (2010). The cultural dimension of sustainability. Religion and Dangerous Environmental Change: Transdisciplinary Perspectives on the Ethics of Climate and Sustainability, 145.

Brugmann, J. (1997). Is there a method in our measurement? The use of indicators in local sustainable development planning. Local Environment, 2(1), 59-72.

Brundtland, C. (1987). World commission on environment and development. Our common future.

Centro de Patrimonio Mundial de la UNESCO (CPM), (2003). World Heritage Papers, No. 6. World Heritage Cultural Landscapes 1992-2002. Recuperado de:http://unesdoc.unesco.org/images/0013/001331/133121e.pdf

Centro de patrimonio Mundial (CPM) Lista de inscripciones de Patrimonio Mundial. Recuperado de: http://whc.unesco.org/en/syndication

De Vries, B. J. M., \& Petersen, A. C. (2009). Conceptualizing sustainable development: An assessment methodology connecting values, knowledge, worldviews and scenarios. Ecological Economics, 68(4), 1006-1019. 
La incorporación de la cultura y el patrimonio ...

Dessein, J., Soini, K., Fairclough, G., \& Horlings, L. G. (2015). Culture in, for and As Sustainable Development; Conclusions from the COST Action IS1007 Investigating Cultural Sustainability: University of Jyväskylä.

Dylan, A. (2012). Rethinking sustainability on planet Earth: A time for new framings. Electronic Green Journal, 1(34).

Galla, A. (2012). World Heritage: benefits beyond borders: Cambridge University Press.

García Canclini, N., \& Criado, A. (1999). Los usos sociales del patrimonio cultural: Consejería de Cultura. Junta de Andalucía.

Grimwade, G., \& Carter, B. (2000). Managing small heritage sites with interpretation and community involvement. International Journal of Heritage Studies, $6(1), 33-48$.

Harrison, R. (2013). Heritage: critical approaches: Routledge.

Hassan, A. M., \& Lee, H. (2015). Toward the sustainable development of urban areas: An overview of global trends in trials and policies. Land Use Policy, 48, 199-212.

Holden, M. (2017). Pragmatic Justifications for the Sustainable City: Acting in the Common Place: Taylor \& Francis.

Hosagrahar, J. (2015). Urban Heritage and Sustainable Development in South Asia: A Plea for a Heritage-Aware Approach Perceptions of Sustainability in Heritage Studies (Vol. 4, pp. 113-124): Walter de Gruyter GmbH \& Co KG.

ICOMOS, (1998). Conferencia Intergubernamental sobre Políticas Culturales para el desarrollo. Recuperado de: http://unesdoc.unesco.org/images/0011/001139/ 113935so.pdf 
Comisión Mundial de Cultura y Desarrollo(CMCD), (1997). Nuestra DiversidadCreativa. Recuperado de: http://unesdoc.unesco.org/images/0010/001036/103628s. pdf

ICOMOS, (1987.) Carta de Washington para la Conservación de Ciudades Históricas y Áreas Urbanas Históricas. Recuperado de: https://www.icomos.org/charters/ towns_sp.pdf

ICOMOS, (1994). Documento de Nara sobre la Autenticidad del Patrimonio Cultural. Recuperado de: http://www.esicomos.org/Nueva_carpeta/info_DOC_NARAesp.htm

ICOMOS, (1999). Carta de Burra para Sitios de Significación Cultural. Recuperado de: http://ipce.mcu.es/pdfs/1999_Carta_de_Burra.pdf

ICOMOS, (2007). Carta Ename sobre Interpretación y Presentación de Sitios de Patrimonio Cultural. Recuperado de: http:/www.enamecharter.org/downloads/ ICOMOS_Carta_Interpretacion_ES.pdf

ICOMOS, 2011, Paris Declaration on Heritage as a Driver of Development, Recuperado de: http://www.international.icomos.org/Paris2011/GA2011_Declaration_de_Paris_EN_20120109. Pdf

International Federation of Arts Councils and Culture Agencies (IFACCA), (2013), Culture as Goal in the Post-2015 Development Agenda. Recuperado de: http:// media.ifacca.org/files/cultureasgoalweb. pdf

Jokilehto, J., Cleere, H., Denyer, S., \& Petzet, M. (2005). The World Heritage List: Filling the Gaps: an Action Plan for the Future: ICOMOS.

Labadi, S. (2017). UNESCO, World Heritage, and Sustainable Development: International Discourses and Local Impacts Collision or Collaboration (pp. 45-60): Springer. 
La incorporación de la cultura y el patrimonio ...

Mišetić. A.. \& Ursić. S. (2015). Remembering cities: the role of memory in the culturally sustainable development of Dubrovnik Theory and Practice in Heritage and Sustainability: Between past and future: Routledge.

Naciones Unidas (ONU). (1992). Conferencia sobre el Medio Ambiente y el Desarrollo (CNUMAD). Recuperado de: http://www.un.org/spanish/esa/sustdev/ agenda21/riodeclaration.htm

Naciones Unidas (ONU). (2000). Resolution 55/2. United Nations Millennium Declaration. Recuperado de:,http://www.un.org/millennium/declaration/ares552e. htm

Naciones Unidas (ONU). (2010). Keeping the promise: united to achieve the Millennium Development Goals - Outcome Document of the 2010 MDG Summit. Recuperado de: http:/www.un.org/en/mdg/summit2010/pdf/mdg\%20outcome\%20document. pdf

Naciones Unidas (ONU). (2012). Conferencia de las Naciones Unidas sobre Desarrollo Sostenible. El futuro que queremos. Recuperado de: https://rio20.un.org/ sites/rio20.un.org/files/a-conf.216-1-1_spanish.pdf.pdf

Naciones Unidas (ONU). (2015). Transformar nuestro mundo: la Agenda 2030 para el Desarrollo Sostenible. Recuperado de: http://unctad.org/meetings/es/sessionaldocuments/ares70d1_es.pdf

Naciones Unidas (ONU). (1988). Decenio Mundial para el Desarrollo Cultural (19881997). A/RES/44/238. Recuperado de: http://www.un.org/en/documents/index. html

Naciones Unidas (ONU). (1991). Creación de la CMCD. A/RES/46/158.. Recuperado de: http://www.un.org/en/documents/index.html

Naciones Unidas (ONU). (1996). Asamblea General sobre el Desarrollo de los Recursos Humanos para el Desarrollo. A/RES/50/105. Recuperado de: http://www. un.org/en/documents/index.html 
Naciones Unidas (ONU). (2002). Año de las Naciones Unidas para el Patrimonio Cultural Mundial. A/RES/56/8. Recuperado de: http://www.un.org/en/documents/index.html

Naciones Unidas (ONU). (2010). Resolución 65/166 sobre Cultura y Desarrollo. A/ RES/65/166. Recuperado de: http://www.un.org/en/documents/index.html

Naciones Unidas (ONU). (2011). Resolución 66/208 sobre Cultura y Desarrollo A/ RES/66/208. Recuperado de: http://www.un.org/en/documents/index.html

Naciones Unidas (ONU). (2013). Resolución 68/223 sobre Cultura y Desarrollo A/ RES/68/223. Recuperado de: http://www.un.org/en/documents/index.html

Naciones Unidas (ONU). (2015). Resolución 70/214 sobre Cultura y Desarrollo A/ RES/70/214. Recuperado de: http://www.un.org/en/documents/index.html

Pezzoli. K. (1997). Sustainable development: A transdisciplinary overview of the literature. Journal of Environmental Planning and management. 40(5). 549-574.

PNUD. 2004. Informe sobre Desarrollo Humano. La Libertad Cultural en el Mundo Diverso de Hoy. Recuperado de: http:/hdr.undp.org/sites/default/files/ hdr_2004_es.pdf

Reid. D. (2013). Sustainable development: an introductory guide: Routledge.

Redclift M. 2005. Sustainable development (1987-2005): an oxymoron comes of age. Sustainable Development 13(4): 212-227

Rodwell. D. (2007). Conservation: Background. Conservation and Sustainability in Historic Cities. 1-22.

Schurman. R. A. (1996). Snails. southern hake and sustainability: neoliberalism and natural resource exports in Chile. World Development. 24(11). 1695-1709. 
La incorporación de la cultura y el patrimonio ...

Soini. K.. \& Birkeland. I. (2014). Exploring the scientific discourse on cultural sustainability. Geoforum. 51. 213-223.

Soini. K.. \& Dessein. J. (2016). Culture-Sustainability Relation: Towards a Conceptual Framework. Sustainability. 8(2). 167.

Stubbs. M. (2004). Heritage-sustainability: developing a methodology for the sustainable appraisal of the historic environment. Planning Practice \& Research. 19(3). 285305. doi: 10.1080/0269745042000323229

Stylianou-Lambert. T.. Boukas. N.. \& Bounia. A. (2015). 11 Politics. tourism and cultural sustainability. Theory and Practice in Heritage and Sustainability: Between Past and Future. 176.

Thomson. I. (2015). 'But does sustainability need capitalism or an integrated report' a commentary on 'The International Integrated Reporting Council: A story of failure' by Flower. J. Critical Perspectives on Accounting. 27. 18-22. doi: http://dx.doi.org/10.1016/j.cpa.2014.07.003

Throsby. D. (2001). Economics and culture: Cambridge university press.

Tweed. C.. \& Sutherland. M. (2007). Built cultural heritage and sustainable urban development. Landscape and Urban Planning. 83(1). 62-69. doi: http://dx.doi.org/10.1016/j.landurbplan.2007.05.008

UNESCO. (1967). Recomendación relativa a la Participación y la Contribución de las Masas Populares en la Vida Cultural. Recuperado de: http://unesdoc.unesco.org/ images $/ 0011 / 001140 / 114038$ s.pdf\#page $=146$

UNESCO. (1972). Convención para la Salvaguarda del Patrimonio Mundial Cultural y Natural. Recuperado de: http://whc.unesco.org/archive/convention-es.pdf

UNESCO. (1976) Recomendación relativa a la Participación y la Contribución de las Masas Populares en la Vida Cultural. Recuperado de: http://portal.unesco.org/es/

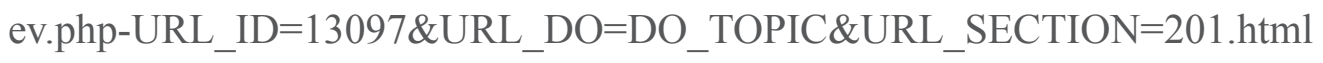


UNESCO. (1976). Recomendación relativa a la Salvaguardia de los Conjuntos Históricos o Tradicionales y su Función en la Vida Contemporánea. Recu-perado de: http://portal.unesco.org/es/ev.php-URL_ID=13133\&URL_DO=DO_TOPIC\&URL_SECTION=201.html

UNESCO. (1982). Conferencia Mundial sobre Las Políticas Culturales (MON-DIACULT). Recuperado de: http://unesdoc.unesco.org/images/0005/000525/052505sb.pdf

UNESCO. (1990). Declaración del decenio mundial para el desarrollo cultural http:// unesdoc.unesco.org/images/0008/000852/085287sb.pdf

UNESCO. (1997). Declaration on the Responsibilities of the Present Generations Towards FutureGenerations. Recuperado de: http://portal.unesco.org/en/ev. php-URL_ID $=13178 \& U R L \_D O=D O \_T O P I C \& U R L \_S E C T I O N=201 . \mathrm{html}$

UNESCO. (2001). Declaración Universal de la UNESCO sobre la Diversidad Cul-tural. Recuperado de: http://unesdoc.unesco.org/images/0012/001271/127162s. pdf

UNESCO. (2002). Declaración de Budapest sobre Patrimonio Mundial. Recupe-rado de: http://www.patrimonio-mundial.com/decl-budapest.pdf

UNESCO. (2003). Convención para la Salvaguardia del Patrimonio Cultural Inma-terial. Recuperado de: http://unesdoc.unesco.org/images/0013/001325/132540s. pdf

UNESCO.( 2005). Convención sobre la Protección y la Promoción de la Diversi-dad de las Expresiones Culturales. Recuperado de: http://unesdoc.unesco.org/images/0014/001429/142919s.pdf

UNESCO.( 2005). Vienna Memorandum on "World Heritage and Contemporary Architecture - Managing the Historic Urban Landscape”. Recuperado de: http:// whc.unesco.org/archive/2005/whc05-15gainf7e.pdf 
La incorporación de la cultura y el patrimonio ...

UNESCO.( 2011). Future of the World Heritage Convention. Recuperado de: http:// whc.unesco.org/archive/2011/whc11-18ga-11-en.pdf

UNESCO. (2011). Recommendation on the Historic Urban Landscape. Recuperado de: http://portal.unesco.org/en/ev. php-URL_ID $=48857 \& U R L \_D O=D O \_T O-$ PIC\&URL_SECTION=201. html

UNESCO. (2011). Strategic Action Plan for the Implementation of the World Heritage Convention 2012-2022. Future of the World Heritage Convention. Document WHC-11/18.GA/11. Recuperado de: http://whc.unesco.org/archive/2011/ whc11-18ga-11-en. pdf

UNESCO.(2012). Visión de Kyoto. Recuperado de: whc.unesco.org/document/123339

UNESCO. (2012b) Convention concerning the protection of the World Cultural and Natural Heritage. World Heritage Committee Thirty-sixth session Saint Petersburg. Russian Federation 24 June- 6 July 2012. Recuperado de: http://whc. unesco.org/archive/2012/whc12-36com-5C-en.pdf

UNESCO. (2012). Culture: a driver and enabler of sustainable development. Thematic Think Peace. Recuperado de: https:/en.unesco.org/post2015/sites/post2015/ files/Think\%20Piece\%20Culture. pdf

UNESCO. (2012). Reports of the World Heritage Centre and Advisory Bodies. World Heritage Convention and Sustainable Development. Document WHC-12/36. COM/5C. Recuperado de: http://whc.unesco.org/archive/2012/whc1236com-5C-en. pdf

UNESCO. (2013). The Hangzhou Declaration - Placing Culture at the Heart of Sustainable Development Policies. Recuperado de: http:/www.unesco.org/new/fileadmin/MULTIMEDIA/HQ/CLT/images/FinalHangzhouDeclaration20130517. Pdf 
UNESCO. (2014). Declaración de Florencia sobre Cultura. Creatividad y Desarrollo Sostenible. Investigación. Innovación. Oportunidades. Recuperado de: http:// unesdoc.unesco.org/images/0023/002303/230394s.pdf

UNESCO. (2015). 20a Reunión de la Asamblea General de los Estados Partes en la Convención para la Protección del Patrimonio Mundial. Cultural y Natural. Patrimonio Mundial y desarrollo sostenible WHC-15/20.GA/13. Recuperado de: http://whc.unesco.org/archive/2015/whc15-20ga-13-es.pdf

United Cities and Local Governments (UCLG). (2008). Agenda 21 for Culture. 8 May 2004. Recuperado de: http://www.agenda21 culture.net/index.php/documents/agenda-21-for-culture

United Cities and Local Governments (UCLG). (2010). Culture: Fourth Pillar of Sustainable Development. Recuperado de: http://www.culturaldevelopment.net. au/wp-content/uploads/2010/11/Culture4pillar-policy.pdf

van Oers. R.. \& Pereira Roders. A. (2012). Historic cities as model of sustainability. Journal of Cultural Heritage Management and Sustainable Development. 2(1). 4-14.

Vlassis. A. (2015). Culture in the post-2015 development agenda: the anatomy of an international mobilisation. Third World Quarterly. 36(9). 1649-1662. doi: 10.1080/01436597.2015.1052064

Walker. E. J.. \& others. (2014). Exploring Socio-Cultural Dimensions of Sustainability. How Cultural and Social Factors Inform a Sustainable Redesign of Whitmore Park (Annapolis. MD)

White. C. (2016). The conditions of practical action: Neoliberalism and sustainability in the Australian road construction industry. Environment and Planning C: Government and Policy. 34(8). 1501-1515.

Williams. R. (2014). Keywords: A vocabulary of culture and society: Oxford University Press. 
Wolfensohn. J. D. (2000). Culture counts: financing. resources. and the economics of culture in sustainable development: proceedings of the conference held in Florence. Italy. October 4-7. 1999. cosponsored by the government of Italy and the World Bank. in cooperation with the United Nations Educational. Scientific. and Cultural Organization.

Wu. S. R.. Fan. P.. \& Chen. J. (2016). Incorporating culture into sustainable development: A cultural sustainability index framework for green buildings. Sustainable Development. 24(1). 64-76.

\author{
Fuentes de financiamiento
}

Esta investigación ha sido financiada por la Secretaría Nacional de Educación Superior Ciencia y Tecnología (SENESCYT) del Ecuador.

\title{
¿Cómo citar este artículo?
}

Molina Neira, B.A. (Enero-junio, 2018). La incorporación de la cultura y el patrimonio en el desarrollo sostenible: desafíos y posibilidades. Revista humanidades, 8(1), 25-50. doi: http://dx.doi.org/10.15517/h.v8i1.31465 